\title{
APERTURE SYNTHESIS IMAGING FROM THE MOON
}

\author{
JACK O. BURNS \\ New Mexico State University, Department of Astronomy, Box \\ 30001/Dept. 4500, Las Cruces, NM 88003
}

\begin{abstract}
Four candidate imaging aperture synthesis concepts are described for possible emplacement on the Moon beginning in the next decade. These include an optical interferometer with $10 \mu \mathrm{arcsec}$ resolution, a submillimeter array with 6 milliarcsec resolution, a MoonEarth VLBI experiment, and a very low frequency interferometer in lunar orbit.
\end{abstract}

\section{INTRODUCTION}

In July of 1989, President Bush proposed an ambitious plan for the American, and possibly the international, space program. This plan has come to be known as the Space Exploration Initiative (SEI). It involves the establishment of a permanently manned Lunar Outpost within the first decade of the 21st century. This base is intended to have a strong scientific component including lunar geoscience stations and advanced astronomical observatories. The lunar outpost would be used as a stepping stone for a manned Mars mission that would be undertaken later in the next century.

It is now generally recognized that the Moon may very well be the best location within the inner solar system from which to site the next generation space telescopes at a variety of wavelengths (e.g., Burns and Wendell 1988). A number of interesting suggestions have been made for advanced astrophysical observations that are well suited to the lunar environment (e.g., Mumma and Smith 1990). Not surprisingly, many of these concepts involve interferometry and aperture synthesis - tools that are easily adaptable to the lunar surface. In this paper, I will first discuss the advantages of the Moon for interferometry. Then, I will describe four classes of candidate lunar-based telescopes that utilize aperture synthesis imaging: an optical interferometer, a submillimeter array, Moon-Earth VLBI, and a very low frequency interferometer operating below $30 \mathrm{MHz}$.

\section{ADVANTAGES OF THE MOON FOR INTERFEROMETRY}

\section{Ultra-high Vacuum}

The average density of the lunar atmosphere is only $2 \times 10^{5}$ molecules $\mathrm{cm}^{-3}$ on the night side. This is considerably less than that of the Earth's atmosphere 
at low Earth orbit. The entire lunar atmospheric mass is $10^{4} \mathrm{~kg}$ - less than that within a typical basketball arena on Earth. The lunar ionospheric density is believed to be $<100$ ions $\mathrm{cm}^{-3}$ which corresponds to a plasma frequency of $<90 \mathrm{kHz}$. Thus, for all but the very lowest frequencies, one can perform diffraction-limited imaging from the Moon. Also, there is no windloading or adverse weather like that on the Earth.

\section{Low Gravitational Acceleration}

The fact that the Moon has some gravity is generally considered an advantage for construction over that of the effective zero-g environment of LEO (e.g., Johnson and Wetzel 1990). However, with gravity only one-sixth that of Earth, the gravitational loading on telescopes is substantially reduced in comparison to the Earth. Therefore, it will be possible to build much larger structures on the Moon. This will substantially enhance the sensitivity of single telescopes and elements of interferometric arrays.

\section{Low Seismicity}

For all intents and purposes, the Moon is a nearly dead world, geologically speaking. Average ground motions are $<1 \mathrm{~nm}$ and the average seismic energy of the Moon is $10^{-8}$ that of the Earth. Furthermore, the seismic waves that are generated by moonquakes do not propagate very far because of damping by subsurface irregularities in the lunar regolith. Thus, the Moon provides an ideal stable surface for long baseline interferometry. No large platforms or costly station-keeping of individual elements is needed as in Earth orbit.

\section{Abundant Real Estate}

Following on the last advantage, the Moon (especially on the near-side) contains abundant large and flat land areas in the marias that are ideally suited for deploying extensive arrays of telescopes.

\section{The Lunar Far-Side}

The lunar far-side is the most important attribute of the Moon for very low frequency radio astronomy. The RAE-2 satellite launched into lunar orbit in the early 1970's unequivocally demonstrated that the far-side of the Moon is the only truly radio-quiet environment in the Earth-Moon system (Kaiser 1990). Substantial ionospheric breakthrough of man-made interference (especially on the terrestrial night-side) makes observations between 5 and $30 \mathrm{MHz}$ very difficult in Earth orbit (Erickson 1990). Below $1 \mathrm{MHz}$, the auroral kilometric radiation (AKR), originating from plasma processes in the Earth's magnetotail, is many orders of magnitude brighter than the brightest extraterrestrial source (Kaiser 1990). Thus, the lunar far-side offers a unique environment in which sensitive high resolution observations of radio sources below $30 \mathrm{MHz}$ can be performed.

\section{Slow Thermal Cycling}

Recent experience with the Hubble Space Telescope demonstrates the difficulties of dealing with thermal instabilities in spacecraft structure in LEO where the spacecraft passes in and out of darkness every 45 minutes. On the Moon, there are much longer, and therefore, more stable periods of uniform heating - the lunar surface experiences two weeks of day and two weeks of 
night. Thus, the slower thermal cycling on the Moon will ease the thermal loading problems on telescope superstructure in comparison to LEO.

\section{LOUISA: LUNAR OPTICAL-UV-IR SYNTHESIS ARRAY}

The concept of a lunar optical interferometer can be traced back to the mid1960's in panel discussions of Phase II Apollo scientific experiments. More recently, Burke (1985) proposed a 27 element optical VLA on the Moon composed of one-meter class elements. This idea was examined in more detail at recent workshops (Burns et al. 1991; Shao 1990). An optical interferometer is well suited to an evolutionary construction strategy in which two elements are initially placed on the Moon. Techniques for calibration, laser metrology, and astrometry can be investigated with a single baseline interferometer. In subsequent trips to the Moon, more optical telescopes can be emplaced to expand the interferometer to an imaging array.

The candidate configuration for the "ultimate" optical-uv-IR array that emerged from the workshop described in Burns et al. (1991) has the acronym LOUISA. This array consists of $421.5-\mathrm{m}$ optical elements distributed in two concentric circles. The outer circle has a diameter of $10-\mathrm{km}$ (limitation imposed by the curvature of the lunar surface) and the inner circle of 9 elements has a diameter of $0.5-\mathrm{km}$. The elements are distributed nonuniformly along both circles. This configuration has superior instantaneous u-v coverage to that of a VLA Y and produces a synthesized beam with much lower sidelobes (Cornwell 1988; Burns 1991). Instantaneous coverage of the u-v plane is more important on the lunar surface because the Moon rotates 27 times more slowly than the Earth, and thus rotation synthesis is not as efficient. However, one problem with using circular arrays for optical interferometry concerns the polarization. Light reflecting from a mirror at an angle will have its two orthogonal polarizations phase shifted relative to each other. Therefore, one must consider in designing a circular array that radial light beam combinations will produce many different phase shifts. The resolution for the proposed array will be $10 \mu \operatorname{arcsec}$ at a wavelength of $0.5 \mu \mathrm{m}$, nearly 100,000 times better than typical ground-based images.

The science that one will perform with LOUISA is very impressive, possibly more so than any other candidate telescope proposed for the Moon. For example, at a distance of $10 \mathrm{pc}$, the Sun and Jupiter would form a pair that is 0.5 apart. Earth would be 0.1 away from the Sun. Jupiter and the Earth would be $\approx 10^{-4}$ arcsec and $\approx 10^{-5}$ arcsec in diameter, respectively. Thus, in principle, a Jupiter-like planet would be well resolved and an Earthlike planet would be partially resolved by LOUISA if these planets are at a distance of a nearby system such as $\tau$ Ceti. However, collecting area and sensitivity are key issues if such an array is to actually image such extrasolar planets. Similarly, LOUISA could resolve active regions on other stars and probe the internal structures of these stars via their surface motions. Another possible project for LOUISA involves detailed measurements of stellar dynamics deep within the cores of galaxies to place limits on the mass of large compact objects. Finally, astrometric measurements of nearby quasar proper motions could be used to study Hubble flow anisotropies." 


\section{SALSA: SYNTHESIS ARRAY FOR LUNAR SUBMILLIMETER}

\section{ASTRONOMY}

The high vacuum and excellent seismic stability make the Moon an excellent location for a future submillimeter array. This idea has been studied by Mahoney (1990). His design calls for an evolutionary scheme that begins with three stations initially operating at $250 \mu \mathrm{m}$. This would eventually expand to six stations operating between 30 and $300 \mu \mathrm{m}$. Each telescope would be $4-5$ $\mathrm{m}$ in diameter. The baselines range from 50 to $1000 \mathrm{~m}$ producing a resolution of $0{ }^{\prime \prime} 006$ at the shortest wavelength. Both continuum and line receivers would be available. Passive cooling could be utilized during the lunar night when temperatures average $\approx 100 \mathrm{~K}$.

The scientific drivers for SALSA include studying regions around protostellar and protoplanetary disks, examining starburst activity in distant galaxies, and measuring the fine scale structure that is expected to exist in the cosmic background radiation.

\section{MERI: MOON-EARTH RADIO INTERFEROMETER}

A natural extension of current international plans for space-based VLBI would be the emplacement of a radio telescope on the Moon to extend baselines to distances of about $384,000 \mathrm{~km}$. Initially, one might perform an experiment analogous to that done with the TDRSS satellite several years ago (Levy et al. 1986). One might use a communication antenna, needed for the Lunar Outpost, to search for fringes for bright compact sources on these long baselines. The $u-v$ coverage for an array that includes the VLBA, VSOP, Radioastron and a lunar antenna is quite reasonable (Burns and Asbell 1987). The resolution would be $13 \mu \operatorname{arcsec}$ at $10 \mathrm{GHz}$.

Such an ultralong baseline experiment would be useful for constraining basic models of compact extragalactic radio sources, relativistic beaming models, and basic radiation physics (synchrotron and inverse Compton scattering).

\section{LORAE: LUNAR ORBIT RADIO ASTRONOMY EXPERIMENT}

The lunar far-side is now recognized as the best location for a future low frequency array (e.g. Burns et al. 1989). However, it will be some time before such construction activities will be possible on the far-side even after the establishment of a near-side outpost. Nonetheless, we can still take advantage of the low noise levels of the far-side and perform high resolution imaging by placing antennas in orbit about the Moon. Plans are evolving for precursor lunar orbiting missions by the U.S. and the Japanese that could carry such low frequency antennas into lunar orbit.

We have proposed to place relatively simple phased arrays of low frequency dipoles on two polar orbiting spacecraft which would then permit us to perform interferometry using the two antennas (Burns 1990). The receivers would operate from $\approx 15 \mathrm{kHz}$ to $10 \mathrm{MHz}$ in a total power mode (making observations of the Earth's AKR as well as the spectral/temporal distribution of interference from Earth; solar bursts; and Jovian planet radio 
emission) and at 13 and $25 \mathrm{MHz}$ in the interferometric mode. We plan to calibrate the baseline by observing point sources at $\approx 150 \mathrm{MHz}$ where the sensitivity of the antennas will be higher. An extensive array of dipoles is required to achieve directivities of 10 at $26 \mathrm{MHz}$ and 100 at $150 \mathrm{MHz}$. So, we are proposing to deploy inflatiable dipoles which are very low mass (array mass is $<1 \mathrm{~kg}$ ) and can accomodate a complex configuration.

Each spacecraft orbits the Moon with a period of $\approx 2$ hrs and their orbits will likely be in perpendicular planes. The resulting range of baselines for LORAE will be between a few $\mathrm{km}$ and $3500 \mathrm{~km}$. However, because of interplanetary scintillation, the maximum useful baseline will be $<1500 \mathrm{~km}$ when viewing sources at large angles to the Sun (even shorter closer to the Sun). Thus, the resolution and field of view for LORAE is expected to be $1-2$ arcsec and $\approx 1^{\prime}$, respectively. This resolution is a significant advance over the best ground-based imaging (at $38 \mathrm{MHz}$ ) by more than two orders of magnitude (Rees 1990).

Simulated $\mathrm{u}-\mathrm{v}$ coverage for LORAE is surprisingly good, as shown in Figure 1. The resulting synthesized beam has sidelobes of only a few percent. A dirty map of an idealized 3C-like triple source observed with LORAE is also shown in Figure 1. It, too, is surprisingly good for a simple two element interferometer. (This map assumes one can deal with the difficulties involved with large field of view mapping.)

The sensitivity for LORAE is adequate. Assuming a directivity of 10 , a modest bandwidth of $50 \mathrm{kHz}$, and an integration time of $10^{6} \mathrm{sec}$, the rms noise will be $<2 \mathrm{Jy}$. This, also, is comparable to the best ground-based imaging sensitivity with more extensive arrays (Rees 1990).

It appears to us that we can quite successfully and simply perform very low frequency aperture synthesis from lunar orbit where the interference levels are greatly reduced (when on the far-side). Such a telescope will achieve resolutions comparable to that of the VLA at $\mathrm{cm}$ wavelengths. With the addition of more spacecraft in lunar orbit, as might be expected as SEI progresses and with the possible involvement of the Japanese and the Soviets, even more extensive and sensitive observations at low frequencies (using closure techniques) might be expected. LORAE will also be an important precursor to future low frequency observatories on the lunar surface (e.g., Douglas and Smith 1985, Burns et al. 1989).

In addition to solar and planetary burst observations, LORAE will investigate the physical conditions in QSOs and AGNs via their low electron energy populations, search for remnants of previous activity epochs in galaxies, and study in-situ particle acceleration in supernova remnants.

\section{SUMMARY}

The recently announced presidential Space Exploration Initiative has given a major boost to the concept of lunar-based observatories. A number of the proposed telescopes will attempt to achieve very high resolution imaging using aperture synthesis techniques. It is not too soon to begin planning these observatories, especially given the long lead times for recent space-based telescope projects. 

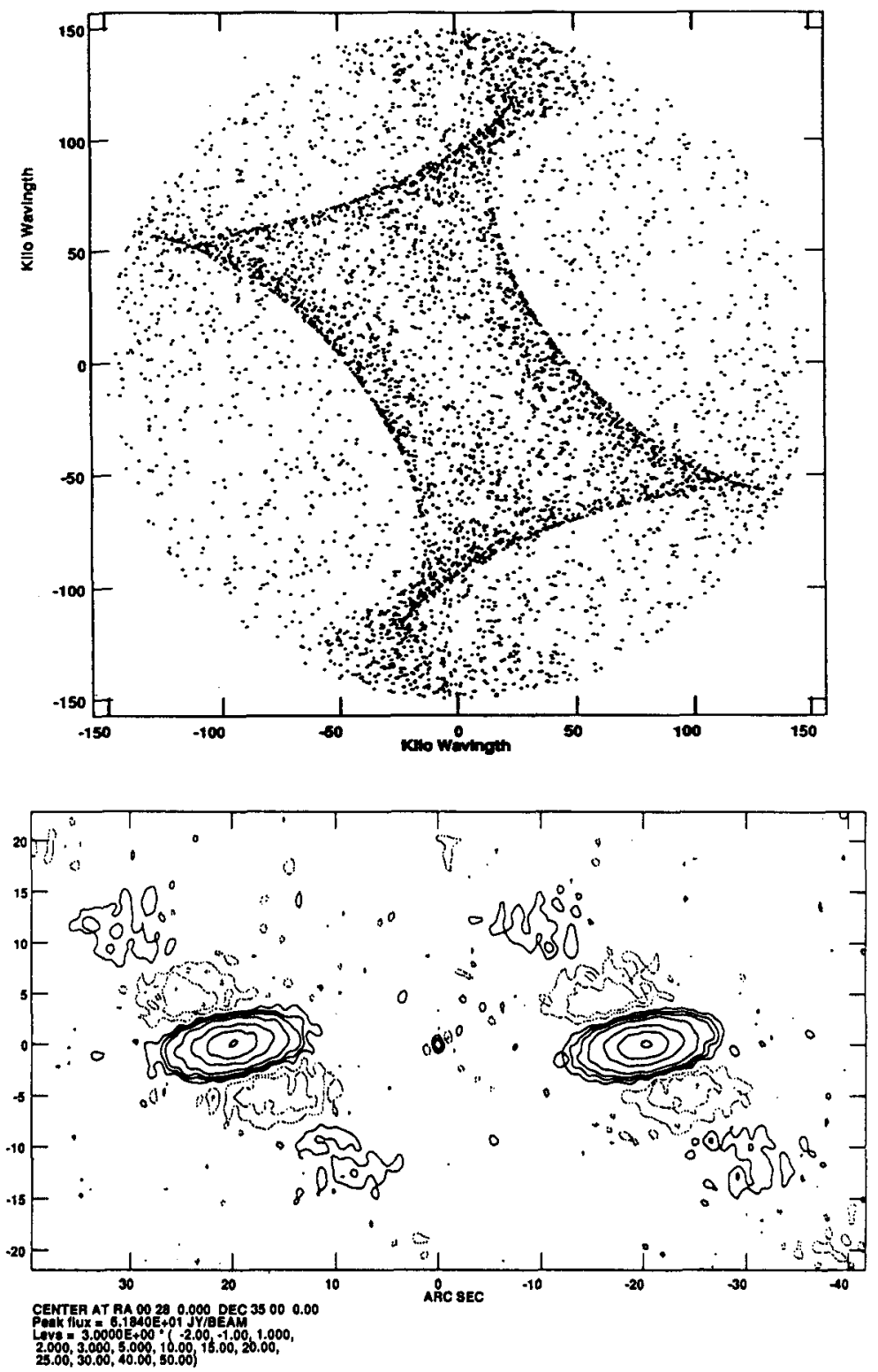

Fig. 1. Top figure is a $\mathrm{u}-\mathrm{v}$ plot for LORAE assuming two satellites in polar orbits separated by $90^{\circ}$, source position at $\alpha=7^{h}$ and $\delta=35^{\circ}$, observations only when both spacecraft are on the far-side, and one-year total integration sampled in $1^{m}$ intervals (but only every 100 th point plotted here). Bottom image is simulated dirty map for a $3 \mathrm{C}$-like triple source. 


\section{ACKNOWLEDGEMENTS}

I would like to thank B. Dennison, J. Basart, N. Duric, S. Johnson, J. Taylor, S. Castillo and the staff of the NMSU Physical Sciences Laboratory for their significant contributions to these lunar telescope concepts. This work was supported by a grant from the NASA Johnson Space Center.

\section{REFERENCES}

Burke, B. 1985, in Lunar Bases and Space Activities of the 21st Century, ed W. Mendell (Lunar and Planetary Inst.: Houston), p. 281.

Burns, J. and Asbell, J. 1987 in Radio Astronomy From Space, ed K. Weiler (NRAO: Green Bank), p. 29.

Burns, J. and Mendell, W. 1988, Future Astronomical Observatories on the Moon, NASA Conference Publication 2489.

Burns, J., Duric, N., Johnson, S., and Taylor, J. 1989, A Lunar Far-Side Very Low Frequency Array, NASA Conference Publication 3039.

Burns, J. 1990 in Low Frequency Astrophysics From Space, ed N. Kassim and K. Weiler, Lectures Notes in Physics 362 (Springer-Verlag: New York), p. 19.

Burns, J., Duric, N., and Johnson, S. 1991, Lunar Optical-UV+IR Synthesis Array, NASA Conference Publication, in press.

Burns, J. 1991 in Space Interferometry: Science and Instrument Architectures, ed S. Kulkarni and M. Shao, in press.

Cornwell, T. 1988, IEEE Trans. Antennas and Propagation 36, 1165.

Douglas, J. and Smith, H. 1985 in Lunar Bases and Space Activities of the 21st Century, ed W. Mendell (Lunar and Planetary Inst.: Houston), p. 281.

Erickson, W. 1990 in Low Frequency Astrophysics from Space, ed N. Kassim and K. Weiler, Lecture Notes in Physics 362 (Springer-Verlag: New York), p. 59.

Johnson, S. and Wetzel, J. 1990, Engineering, Construction, and Operations in Space $I$ (ASCE: New York).

Kaiser, M. 1990 in Low Frequency Astrophysics from Space, ed N. Kassim and K. Weiler, Lecture Notes in Physics 362 (Springer-Verlag: New York), p. 3.

Levy, G. et al. 1986, Science 234, 187.

Mahoney, M. 1990 in Astrophysics from the Moon, ed M. Mumma and

H. Smith, AIP Conference Proceedings 207, p. 508.

Mumma, M. and Smith, H. 1990 Astrophysics from the Moon, AIP

Conference Proceedings 207.

Rees, N. 1990, M.N.R.A.S., 244, 233.

Shao, M. 1990 in Astrophysics from the Moon, ed M. Mumma and H. Smith, AIP Conference Proceedings 207, p. 486. 
D. W. Murphy: What is the projected cost for a project such as Louisa? J. O. Burns: It is really impossible to project a cost at the present time. Our working assumption has always been that such lunar-based telescopes will only exist if a well-established infrastructure is emplaced on the Moon and a regular transportation system between the Earth and the Moon is in place. Then, lunar observatories, utilizing this infrastructure (including engineers, labs, power generators) could be built at a "reasonable" price. Certainly, a project like LOUISA will be cheaper and easier to build on the Moon than as free-flyers in Earth orbit.

Goran Pilbratt: A comment and a question. If you perform an Earth-Moon VLBI experiment, and you want to fill in the uv coverage, you need VLBI orbiters at the same time. It is not good enough to use "old" (e.g. VSOP or RADIOASTRON) data because the compact sources are time variable. My question is: Apart from the fact that 42 is the known answer to the question about life, the Universe and everything; how did you perform the trade-off between the number of elements and the size of each element in the case of LOUISA?

J. O. Burns: First, my assumption is that satellites comparable to VSOP and Radioastron will be in orbit when the lunar outpost is established. So, data will be taken simultaneously with the VLBA, the lunar telescope, and these orbiting antennas. Second, no detailed study of the trade-offs between the number of elements and size of the elements has yet been performed for LOUISA. Such studies will need to be performed to optimize the array. Our preliminary modelling involved a plausible guess in terms of the number of elements and their size. A good case can be made for increasing the aperture size for better sensitivity. 